\title{
Credibility Strategies of Popular Health Websites: A Rhetorical Analysis of Parkinson's Information Pages
}

\author{
ABIGAIL BAKKE \\ Department of English \\ Minnesota State University, Mankato \\ Armstrong Hall 230 \\ Mankato, MN 56003 \\ abigail.bakke@mnsu.edu
}

\begin{abstract}
Though using e-health is a common patient practice, unregulated and inaccurate online medical content can pose real dangers. To effectively convey important and accurate health messages, online health organizations must make their information stand out as trustworthy. To identify credibility strategies, I conducted a rhetorical analysis of Parkinson's disease content from the two most popular U.S. health websites: NIH.gov and WebMD.com. Based on the range of credibility strategies identified in these webpages, I recommend that medical writers and e-health designers convey both expertise and trustworthiness to build credibility in their content.
\end{abstract}

KEYWORDS: credibility, e-health, expertise, health, medicine, rhetoric, trust, trustworthiness, visual rhetoric

\section{INTRODUCTION}

Many patients go online to find medical information and support, accessing a broad array of website types, ranging from sources that have been traditionally considered credible, such as governmental or academic sites, to those that build credibility in newer ways, such as patient blogs and social networking sites. Research has shown that many e-health users trust personal, social, and experiential aspects of websites, such as patient narrative and discussion forums (Hether, Murphy, \& Valente, 2014; Sillence \& Briggs, 2015). These patient experience features can be empowering and reassuring for patients. However, situations such as the antivaccination controversy reveal potential dangers in trusting user-generated medical content at the expense of scientifically accurate content.

In order for professional online health organizations to make their important, accurate health messages stand out as trustworthy to users, they need to understand the myriad ways trust is built in e-health. The goal of this paper is to describe the existing strategies in use by the most popular e-health sites, so that researchers can understand the status quo when it comes to e-health credibility, and so that professional health organizations can expand their repertoire of credibility strategies to draw more audiences to their quality information. Therefore, this paper addresses the following question: What credibility strategies do the most popular health websites use? To answer this question, I rhetorically analyzed medical information pages on NIH.gov and WebMD.com, focusing on the multiple ways credibility might manifest in the text, from scientific to personal ways. In this paper, I will first describe the theoretical framework of information credibility that informs my analysis, then I will describe my methods for selecting and analyzing texts. In the findings section, I detail three categories of credibility strategies - writing style, visuals, and citation - and compare how they are deployed across the two websites. I conclude with considerations for researchers and medical writers. 


\section{INFORMATION CREDIBILITY}

To inform my analysis, I turned to the interdisciplinary work on information credibility, which has been studied in fields like mass communication and psychology. While notions such as Aristotle's ethos are applicable, my interest for the purposes of this paper was in the credibility in the medical information pages themselves. ${ }^{1}$ The focus on medical information pages also reflects that internet users typically access medical information through a search engine rather than by starting at a single e-health website (Fox \& Duggan, 2013). Users, then, make quick credibility evaluations based on the content, the visuals, and the formatting and navigability of the page. The research on information credibility predates the internet as we know it today, but nonetheless consists of enduring concepts that apply to modern webpages.

The topic of information or source credibility began to receive special attention surrounding WWII, in an effort to better understand propaganda (Hovland, Janis, \& Kelley, 1953). Out of this time period emerged a definition of credibility that is still generally accepted, that credibility consists of two qualities: expertise (knowledge, competence, intelligence, etc.) and trustworthiness (warmth, sincerity, reliability, etc.). This two-part understanding has been used in various fields, including communication (Metzger \& Flanagin, 2013), computer science (Fogg \& Tseng, 1999), and technical and professional communication (Mackiewicz, Yeats, \& Thornton, 2016). These dimensions of credibility have also been used in psychology to show that the public has tended to respect scientists as experts but does not necessary trust them as warm and sincere (Fiske \& Dupree, 2014).

These studies across disciplines have helped to show the importance of not only more traditional expertise features but also a personal connection - the Fiske and Dupree (2014) study, especially, highlights the need for a multidimensional understanding of credibility in order to help close the gap of trust between scientists and the public. It stands to reason that official health organizations, whether medical institutions or governmental health agencies, could also benefit from a fuller understanding of the credibility strategies that matter to their audiences. These organizations already have the expertise of the medical establishment behind them, but they may fall short in other ways.

\section{METHODS}

I used the method of rhetorical analysis, commonly used in the field of technical communication, to better understand how credibility operates in medical webpages. Rhetorical analysis involves applying elements of rhetorical theory to understand how persuasion operates in texts, with texts construed broadly to include visual and multimodal elements. It closely examines "word choice, arguments, warrants, claims, motives, and other purposeful, persuasive features of language, visuals, and various artifacts" (Lay, Gurak, Gravon, \& Myntti, 2000 , p. 7). For this analysis, I applied the literature reviewed above, which asserts that expertise and trustworthiness are both important elements in establishing credibility.

I chose NIH.gov and WebMD.com to analyze, which are the most popular U.S. health websites according to data from the Alexa Global Traffic Rank (2014). Though these are not

1 Elsewhere, I have explored the organizations' overall ethos as constructed in web design and About pages (Bakke, 2017). It would be valuable to further research how an organization's reputation or ethos interacts with the credibility features of the information itself. 
necessarily the most credible or best health sites, they do best represent the public's current understanding of e-health. The NIH (National Institutes of Health) is the U.S. medical research agency and the largest source of medical research funding in the world. Its website, NIH.gov, serves as a source of information about the institutes' history, mission, and research agenda. The site also serves as a portal to governmental medical literature. WebMD.com is a commercial website, with upwards of 150 million unique visitors per month (WebMD Health Corp, 2014), and has been considered a favorite for many e-health users since the early 2000s (Fox \& Rainie, 2002). It is perhaps best known as a medical information site, publishing news and reference articles on a wide range of health topics.

I chose two sites so that I could document a range of common credibility strategies in health websites. I am particularly interested in the features that map to the two major criteria for credibility: expertise and trustworthiness. With only two websites, I am not able to generalize about how all e-health websites signal their credibility. I also took care to limit my assumptions about whether the organizations would use one type of strategy more than another, so I searched across both sites for scientific credibility markers as well as more personal features that would help communicate the sincerity and warmth of the source.

As for the topic of the webpages, the singular condition of Parkinson's disease allowed for some consistency across the artifacts for analysis. In addition, Parkinson's is the type of condition that would draw many patients and caregivers to e-health for answers: It is a chronic, neurodegenerative disorder that affects approximately a million Americans, and diagnosing and treating it effectively can be a challenge (Parkinson's Disease Foundation, 2015).

Both NIH.gov and WebMD.com have multiple resources pertaining to Parkinson's. The webpages analyzed represent a logical starting point for someone researching the condition (overview-type pages), and then two more pages a click or two away, to account for the fact that e-health users tend to explore beyond a single page. ${ }^{2}$

\section{ANALYSIS RESULTS}

This analysis focuses only on three categories of credibility strategies - writing style, visuals, and citation - in which the websites used noticeably different strategies. NIH.gov clearly displays its connection to standard, trusted procedures for generating medical knowledge, such as clinical trials, while WebMD.com uses a more visual and accessible style and cites more informally, while also using more personal elements to connect with the audience. Nevertheless, given the popularity of both websites, even these different approaches could both be considered rhetorically successful. This results section offers snapshots of how each website approaches credibility in these categories.

2 Specifically, the NIH.gov pages consisted of PubMed Health Parkinson's glossary, overview, and research reviews abstracts page; the WebMD pages consisted of the Parkinson's Health Center, Visual Guide to Parkinson's, and "Understanding Parkinson's" article. 


\subsection{Writing Style}

Writing style can signal credibility in different ways. Jargon, for instance, can signal the author's membership in a specialized, expert field, thereby establishing the expertise component of credibility. At the same time, jargon can cause a reader to feel distanced and illequipped to verify the information, thereby damaging the warmth and sincerity component of credibility. Conversely, overly simplistic language, while accessible, can also come across as condescending. Furthermore, different readers have different knowledge levels and purposes, so in the interest of drawing in a larger audience, a balanced writing style makes sense.

On both NIH.gov and WebMD.com, the writing style represented a balanced, educational tone, but with NIH.gov's language somewhat more distanced, and WebMD's style somewhat more accessible and personal. The following examples represent the basic approaches.

On NIH.gov, the glossary entry for Parkinson's is primarily textual. It defines Parkinson's disease as "A progressive disorder of the nervous system marked by muscle tremors, muscle rigidity, decreased mobility, stooped posture, slow voluntary movements, and a mask-like facial expression," and then cites the source of the definition, the NIH National Cancer Institute. The text, at times, includes some technical terms that might be hard to understand, but provides multiple ways for readers to learn them. For instance, there is a hyperlink from the word "tremors" to a page defining tremors, and at the bottom of the page is a glossary briefly defining other terms to know, including "bradykinesia" and "dyskinesia." At times the textual description includes definitions within the text, for instance, "Parkinson's is a progressive disease, which means that in most cases it will continue to gradually get worse." Overall, the writing style balances medical jargon with explanations for lay users.

WebMD.com's information is much more visual than the NIH information. There is a rotating slideshow of featured articles on the main page. In addition, one of the most prominent resources is "A Visual Guide to Parkinson's Disease," which is a slideshow with 24 images and corresponding information. The slideshow serves as a broad overview of much of the information found in the text-based articles on the site. For instance, there are slides about symptoms, early signs, progression, and diagnosis. Some of the slide titles are phrased as questions, such as "Who gets Parkinson's?" and "Can symptoms be prevented?" The information is quite accessible when broken up and accompanied by visuals; furthermore, the phrasing of headings as questions serves as an invitation to readers to engage with the material.

Another WebMD article illustrates a similar accessible style, though more text-based than the Visual Guide. The article "Understanding Parkinson's Disease: The Basics" opens with "Parkinson's disease mostly affects older people but can also occur in younger adults. The symptoms are the result of the gradual degeneration of nerve cells in the portion of the midbrain that controls body movements. The first signs are likely to be barely noticeable..." The writing style is personal and accessible, in that it places more emphasis on the people Parkinson's affects and how it does so, rather than on the origin or definition of the disease. That being said, the writing does include some more specialized language, such as an explanation of basal ganglia further below. The article includes headings, bullet points, and hyperlinks, much like the NIH information, but with links to more general resources elsewhere on WebMD rather than to more detailed scientific information. The contrast in visuals on both websites led me to more thoroughly analyze and compare visuals as a credibility strategy. 


\subsection{Visuals}

Like the use of writing style, the use of visuals can also appeal to credibility in different ways. Visuals can be more or less technical, and can be deployed for purely informational purposes or to help establish a tone on the webpage. Different types of visuals appeared in each website, contributing to a more expert or a more personal form of credibility - scientific imagery and educational illustrations in NIH.gov, quasi-scientific imagery and humanistic images in WebMD.com.

On the NIH.gov webpages, the main visual is an illustration of a neuron with parts labeled (Fig. 1). The visual is not interactive, but one can find a fuller explanation of neurons by clicking on it. The graphic conveys intricacy while at the same time familiarity, as it resembles the type of illustration found in an anatomy textbook.

\section{Part of: Movement Disorders}

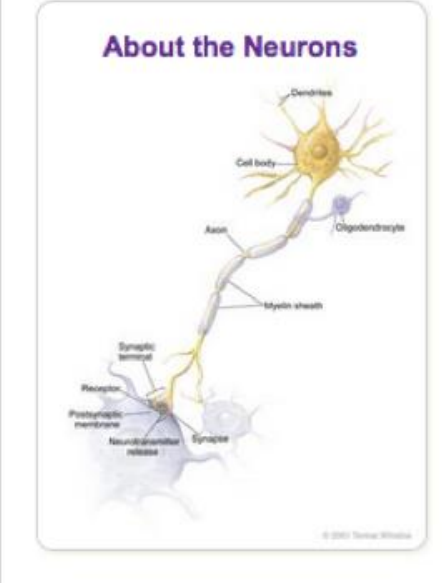

\section{About Parkinson Disease}

Parkinson's is a disease of the nervous system that mostly affects older people. It typically begins after the age of 50 . The disease can be very hard to live with because it severely restricts mobility and as a result makes daily activities increasingly difficult.

Parkinson's is a progressive disease, which means that in most cases it will continue to gradually get worse. Many people who develop Parkinson's will require nursing care. There is no cure for the disease and its exact cause is not known, but there are effective treatments that can relieve the symptoms.

Symptoms

The signs of Parkinson's can be very different. The typical symptoms are:... Read more about Parkinson Disease

Fig. 1. NIH.gov PubMed Health glossary entry for Parkinson's.

On WebMD.com, some of the graphics are scientific, such as computer-generated images of the brain or of the chemical structure of dopamine. Although these graphics are visually appealing, three-dimensional with bright colors, they are not integrated with the text in the standard manner for technical writing. There are no captions, labels, or scientific explanations of the visuals in the text. This means that WebMD.com's visual information is perhaps borrowing the scientific feel of biomedical graphics, but without the corresponding depth.

Most of the non-scientific graphics in the slideshow are generic stock photos connected in some way to the information. There are several images of people in daily life, such as an older man holding a cup of tea, in connection with the slide on demographics (See Fig. 2). Another image is of Michael J. Fox, a well-known actor and spokesperson for Parkinson's, in connection with the slide describing Parkinson's progression. This use of people-based imagery affirms that patients are the central audience.

Although the visual nature of WebMD.com increases the engagement and accessibility of the information, the commercialism can also be distracting. Fig. 2, with a pharmaceutical ad in the bottom right corner, shows the visual prominence of advertisements in WebMD.com 
content. Advertising, understandably, tends to decrease trust in a website (Fogg \& Tseng, 1999).

\section{Slideshow: A Visual Guide to Parkinson's Disease}

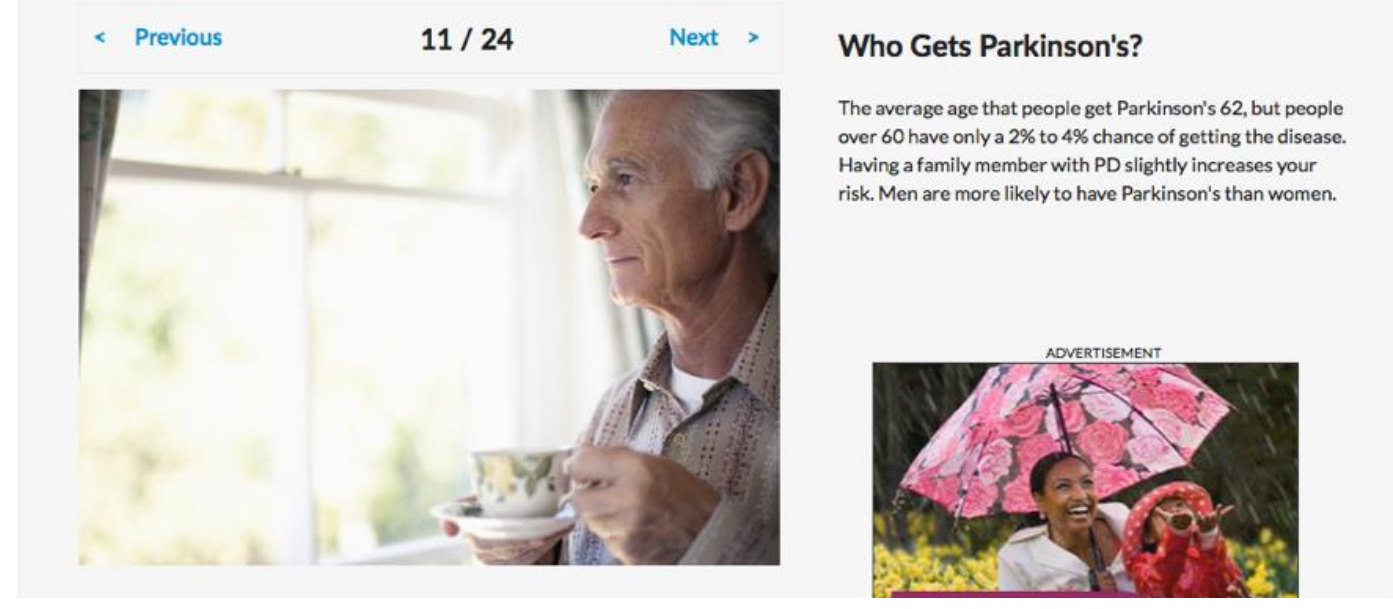

Fig. 2. Example slide in WebMD Visual Guide to Parkinson's Disease.

\subsection{Citation}

Any basic credibility checklist includes the criterion of authorship - the author's identity, credentials, and potential bias. Citation is a significant strategy for conveying credibility, since it enables readers to trace the information back to its original authors. NIH.gov and WebMD.com both used citation to medical experts as a strategy, but in different ways. NIH.gov includes full citations to clinical trials, showing how science is valued as the authority for medical information. WebMD.com includes partial citations to medical organizations, and links to the profiles of physician reviewers, showing the authority accorded to physicians.

One of the sections of NIH.gov's Parkinson's webpages is labeled "What works? Research summarized." There are links to both "Evidence reviews" $(n=155)$ and "Summaries for consumers" ( $n=37)$, several of which have highly technical titles, such as "Pergolide versus bromocriptine for levodopa-induced complications in Parkinson's disease." This section of review studies points to the value this organization places on establishing a baseline of accepted knowledge for treating Parkinson's. This knowledge is built up incrementally, as more and more methodologically-sound studies are conducted regarding treatment efficacy. The participants in these various trials add up to thousands. The assumption is that more research over time provides greater confidence in claims of clinical effectiveness. There is no information about newer, untested therapies. At the bottom is a list of sources, which includes studies published in well-recognized medical journals such as British Medical Journal and Journal of the American Medical Association, thereby invoking the credibility of these major medical publications.

WebMD.com also enables readers to trace the information back to its source, but not as completely as on NIH.gov. Within the Parkinson's webpages, clicking on "View Article Sources" drops down a list of references. Incomplete citation information, however, makes it 
difficult to link the information to specific studies or scientists. For instance, just the organization or journal is listed, such as Journal of the American Medical Association. In addition to peer-reviewed scientific sources such as JAMA, WebMD cites web-based sources authored by organizations, such as the Parkinson's Disease Foundation. Such sources may be more focused on outreach for Parkinson's patients and fund-raising to support research initiatives. Unlike the NIH, which derives its information directly from clinical trials, WebMD draws information from more superficial sources in terms of scientific rigor.

Another citation strategy of WebMD is to list an expert reviewer. For instance, the "Understanding Parkinson's" article states at the bottom, "Reviewed by [Firstname Lastname], MD on February 28, 2015." The Visual Guide likewise lists sources and a reviewer, [Firstname Lastname], MD, MPH," as well as a link to her biography describing her expertise in more depth. The sparse citation information in combination with the stamp of approval from a physician suggests that, when establishing the credibility of its information, WebMD.com appeals more to the authority of physicians than to the authority of scientific research directly.

\section{CONCLUSION}

A patient researching Parkinson's disease would come away from each website with the same general understanding of the disease: who gets it, how it works in the body, and what symptoms and treatments are typical. Furthermore, both websites are relatively traditional, in that they are both unidirectional - they convey information to an audience, rather than creating opportunities for the audience to talk back or to share their own experiences. And while writing style, visuals, and citation convey the credibility of each organization, these categories operate differently for each site, in ways that generally map to the two-part understanding of credibility discussed above: expertise and trustworthiness.

The NIH information conveys credibility in very familiar, traditionally successful ways for medical and scientific discourse: It uses somewhat distanced, technical language, technical visuals, and thorough citations to peer-reviewed medical journals. The technical terminology and visuals convey the knowledge of the source, but the explanations of the terminology also convey trustworthiness in that they help the reader understand. The NIH also appeals to expertise by making the science underlying the information transparent.

WebMD.com likewise cues its expertise with technical terminology, visuals, and physician profiles, but balances those features with more personal ones, such as images of people and more emphasis on the user-friendliness of the information than on its scientific origin. Overall, in comparison to NIH medical information, WebMD's information is clearly more superficial; this is not necessarily problematic, since summary and breadth may be what a user needs rather than details and depth. Though WebMD lacks the depth of NIH information, its scope is broader in a sense. The inclusion of human-interest oriented material suggests that Parkinson's patients are interested in more than just which treatments have been clinically proven to be most effective; they may also be interested in which celebrities share their diagnosis or how they will cope with daily issues like impaired driving skills. The overviewlike nature of the information and explicit listing of medical doctors as expert reviewers show that this information is presented to users as though a doctor might explain a condition to a patient.

It is unsurprising that a governmental health site and a commercial health site would tend towards expertise and trustworthiness markers, respectively, as means to build credibility. 
I do not intend to generalize that governmental e-health sites are always more technical and commercial e-health sites are always more personal, or that one type of site is better than the other. ${ }^{3}$ Warnick (2007) has argued that we view web credibility in terms of field dependence (in Toulmin's terms) - the notion that users evaluate credibility based on that website's context/field and that field's values (e.g., a user would apply different credibility standards for an entertainment site versus a medical site). The present analysis illustrates how the values that underlie credibility vary even within the same "field" - e-health. This is evident in the ways in which Parkinson's information, though essentially the same in content, is presented differently across the two health websites. Audiences may value one type of site over the other depending on their purpose: One patient might appreciate the data underlying medical recommendations, while another patient might want concise, actionable information. Given the diversity of readers, medical writers should profile their readers to best meet their needs. Audience analysis and usability research, tools used by technical communicators, would be of great use to medical writers and e-health designers. These tools would enable medical writers to understand users' goals and preferences in concrete detail, and to improve their medical content accordingly.

Notably, both sites presented a blend of expertise and trustworthiness strategies to build credibility. While it may seem that the participatory web has been drawing all websites in the direction of greater participation by lay users, medical and scientific expertise are still prominent even in more personal or patient-centered websites such as WebMD.com. That being said, these expertise-based strategies can also be appropriated in superficial or misleading ways in order to spread pseudoscience. Therefore, commercial health websites (or any website with accurate medical information derived from scientific data) might adopt strategies such as those that NIH.gov uses in order to amplify the expertise behind the information. Medical writers might even take advantage of hyperlinks, menus, and multiple versions of information in order to tailor the patient's experience. For instance, an article might provide just actionable, personally relevant information for users who desire that, but with links to satisfy other users' desire for depth and transparency. The more that health organizations signal their expertise in transparent ways, the more they will enhance their overall credibility by showing respect for the audience.

Social and personal features indeed have their place too, and as the opening to this paper discussed, users are increasingly trusting such features. Organizations tasked with creating high-quality health content might consider incorporating more patient experience features into their websites to appeal to credibility in broader ways than just traditional scientific means. For medical writers, simply migrating unidirectional textbook-like content from print to digital is not enough to engage and persuade audiences. Rather, medical writers should leverage the interactivity of the web to better communicate not just their expertise but also their trustworthiness; There is more room for creativity, visuals, and patient stories in websites than in print media.

This paper has documented how writing style, visuals, and citation strategies in health websites can convey credibility by appealing to expertise or to trustworthiness (and often

3 This analysis of just two health sites reveals only a subset of the credibility strategies available (albeit the most familiar ones, given each site's huge readership). A valuable next step would be to research user-generated ehealth to see how credibility operates there - presumably via personal, social, and experiential features. 
both). Whatever the website type, medical writers must consider how both components of credibility are needed in order to provide accurate information that audiences also trust.

\section{REFERENCES}

Bakke, A. (2017). Ethos in e-health: From informational to interactive websites. In M. Folk \& S. Apostel (Eds.), Establishing and evaluating digital ethos and online credibility (pp. 85-103). Hershey, PA: IGI Global.

Fiske, S. T., \& Dupree, C. (2014). Gaining trust as well as respect in communicating to motivated audiences about science topics. Proceedings of the National Academy of Sciences, 111(Supplement 4), 13593-13597. https://doi.org/10.1073/pnas.1317505111

Fogg, B. J., \& Tseng, H. (1999). The elements of computer credibility. In Proceedings of the SIGCHI conference on Human Factors in Computing Systems (pp. 80-87). ACM. Retrieved from: http://dl.acm.org/citation.cfm?id=303001

Fox, S., \& Duggan, M. (2013). Health online 2013. Washington, D.C.: Pew Internet and American Life Project. Retrieved from: http://www.pewinternet.org/2013/01/15/health-online-2013/

Fox, S., \& Rainie, L. (2002). Main report: The search for online medical help. Washington, D.C.: Pew Internet and American Life Project. Retrieved from: http://www.pewinternet.org/2002/05/22/main-report-the-search-for-online-medical-help/

Hether, H. J., Murphy, S. T., \& Valente, T. W. (2014). It's better to give than to receive: The role of social support, trust, and participation on health-related social networking sites. Journal of Health Communication, 19(12), 1424-1439. https://dx.doi.org/10.1080/10810730.2014.894596

Hovland, C. I., Janis, I. L., \& Kelley, H. H. (1953). Communication and persuasion: Psychological studies of opinion change. New Haven, CT: Yale University Press.

Lay, M. M., Gurak, L. J., Gravon, C., \& Myntti, C. (Eds.). (2000). Body talk: Rhetoric, technology, reproduction. Madison, WI: University of Wisconsin Press.

Mackiewicz, J., Yeats, D., \& Thornton, T. (2016). The impact of review environment on review credibility. IEEE Transactions on Professional Communication, 59(2), 71-88. https://doi.org/10.1109/TPC.2016.2527249

Metzger, M. J., \& Flanagin, A. J. (2013). Credibility and trust of information in online environments: The use of cognitive heuristics. Journal of Pragmatics, 59, Part B, 210-220. https://doi.org/10.1016/j.pragma.2013.07.012

Parkinson's Disease Foundation. (2015). What is Parkinson's disease? Retrieved from: http://www.pdf.org/about_pd

Sillence, E., \& Briggs, P. (2015). Trust and engagement in online health: A timeline approach. In S. S. Sundar (Ed.), The handbook of the psychology of communication technology (pp. 469-487). Chichester, UK: Wiley Blackwell.

Top 15 most popular health websites. (2014, September). Retrieved from: http://www.ebizmba.com/articles/health-websites

Warnick, B. (2007). Rhetoric online: Persuasion and politics on the World Wide Web (Vol. 12). New York, NY: Peter Lang.

WebMD Health Corp. (2014, February 10). WebMD announces preliminary fourth quarter and full year 2013 results. Retrieved from: http://investor.shareholder.com/wbmd/releasedetail.cfm?ReleaseID=824582\&CompanyID=WBMD 